\title{
Gas-filled Microbubbles - A Novel Susceptibility Contrast Agent for Brain and Liver MRI
}

\author{
April M. Chow, Jerry S. Cheung, Ed X. Wu ${ }^{*}$
}

\begin{abstract}
Gas-filled microbubbles have the potential to become a unique intravascular MR contrast agent due to their magnetic susceptibility effect, biocompatibility and localized manipulation via ultrasound cavitation. However, in vivo demonstration of microbubble susceptibility effect is limited so far and microbubble susceptibility effect is relatively weak when compared with other intravascular MR susceptibility contrast agents. In this study, two types of microbubbles, custom-made albumin-coated microbubbles (AMBs) and a commercially available lipid-based clinical ultrasound contrast agent (SonoVue $\left.{ }^{\circledR}\right)$, were investigated with in vivo dynamic brain and liver MRI in Sprague-Dawley rats at 7 Tesla. Transverse relaxation rate enhancements $\left(\Delta R_{2}{ }^{*}\right)$ maps were computed for brain and liver, yielding results similar to those obtained with a common MR blood pool contrast agent. These results indicate that gas-filled microbubbles can serve as an intravascular MR contrast agent at high field. Enhancement of microbubble susceptibility effect by entrapping monocrystalline iron oxide nanoparticles (MIONs) into microbubbles was also investigated at $7 \mathrm{~T}$ in vitro. This is the first experimental demonstration of microbubble susceptibility enhancement for MRI application. This study indicates that gas-filled microbubble susceptibility effect can be substantially increased using iron oxides nanoparticles. With such approach, microbubbles can potentially be visualized with higher sensitivity and lower concentrations by MRI. Such capability has the potential to lead to real-time MRI guidance in various microbubble-based drug delivery and therapeutic applications.
\end{abstract}

\section{INTRODUCTION}

$\mathrm{G}$ as-filled microbubles can potentially be used as an intravascular MR susceptibility contrast agent in vivo due to the induction of large local magnetic susceptibility difference by the gas-liquid interface. Moreover, microbubbles can be locally cavitated and destroyed by focused ultrasound [1], hence the MR signals can be temporally and spatially manipulated because microbubble disappearances will diminish the susceptibility effect.

Recently, microbubbles have been employed in therapeutic applications due to their unique cavitation [2] and sonoporation [3] properties. Site-specific release of incorporated drugs or genes inside microbubbles can be achieved with local microbubble cavitation by spatially focused ultrasound. Microbubble-mediated sonoporation can dramatically increase cell permeability and intracellular uptake [4], with substantial increase in gene transfection efficiency and therapeutic effectiveness being demonstrated.

Manuscript received on April 7, 2009

This work was supported in part by the University of Hong Kong CRCG grant and Hong Kong Research Grant Council grant.

Ed X. Wu, April M. Chow and Jerry S. Cheung are with the Laboratory of Biomedical Imaging and Signal Processing and the Department of Electrical and Electronic Engineering, The University of Hong Kong, Pokfulam, Hong Kong (phone: (852) 2819-9713; e-mail: ewu@eee.hku.hk).
Furthermore, several therapeutic interventions, like sonothrombolysis [5], transient opening of blood-brain barrier potentially for delivery of both low and high molecular weight therapeutic compounds [6] and enhancing the high intensity focused ultrasound therapy by increasing the local heating rate [7] have been demonstrated.

Early experiment with Albunex ${ }^{\circledR}$, an ultrasound contrast agent consisting of air-filled microbubbles with human albumin shell, illustrated the potential of air-filled microbubbles as an MR susceptibility contrast agent [8]. Feasibility of microbubbles as an MR pressure sensor, based on the susceptibility change caused by pressure-induced microbubble size change, has been explored through theoretical [9] and phantom [10] studies. The first in vivo investigation of susceptibility contrast induced by microbubbles was reported by our group previously using Optison ${ }^{\circledR}$, microbubbles of human albumin shells with perfluorocarbon as core gas, at $7 \mathrm{~T}$ in rat liver [11]. $R_{2}{ }^{*}$ dependency on microbubble volume fraction was also reported for Levovist ${ }^{\circledR}$, air-filled microbubbles with palmitic acid shells, through an in vitro phantom study at $1.5 \mathrm{~T}$ [12]. Magnetic susceptibility enhancement induced by gas-liquid interface was demonstrated recently by simulations and MR experiments using air-filled cylinders in water [13], consolidating the feasibility of gas-filled microbubbles as an MR susceptibility contrast agent. However, in vivo demonstration is limited so far and microbubble susceptibility effect is still relatively weak when compared with other intravascular MR susceptibility contrast agents $[11,14]$. In this study, we aim to further investigate and demonstrate the in vivo MR susceptibility effect induced by both custom-made albumin-coated microbubbles and commercially available lipid-based microbubbles in rat brain and liver at $7 \mathrm{~T}$ using dynamic susceptibility weighted MRI, and experimentally demonstrate that microbubble $R_{2}{ }^{*}$ can be enhanced by embedded or entrapping monocrystalline iron oxide nanoparticles (MIONs) [15] into microbubble shells.

\section{Methodology}

All MRI experiments were performed on a 7 T MRI scanner with a maximum gradient of $360 \mathrm{mT} / \mathrm{m}(70 / 16$ PharmaScan, Bruker Biospin GmbH, Germany). All animal experiments were approved by the local institutional animal ethics committee.

\section{A. Microbubble Preparation}

A commercially available clinical ultrasound contrast agent (SonoVue ${ }^{\circledR}$, Bracco, Milan, Italy), was used in this study. SonoVue ${ }^{\circledR}$ microbubbles consist of sulphur hexafluoride gas stabilized in an aqueous dispersion by a phospholipid monolayer. Air-filled custom-made albumin-coated microbubbles (AMBs) were produced by sonication as 
previously described [16]. AMBs with MIONs embedded in shells were synthesized [17]. Polymeric microbubbles (PMBs) with MIONs incorporated or entrapped in shells were produced by adapting a double emulsion procedure [17]. MION-free PMBs were synthesized with the same procedure except using deionized water instead of the MION solution.

\section{B. Animal Preparation and Microbubble Administration}

Ten normal adult Sprague-Dawley rats ( 250-350 g) were used. Each rat was anesthetized with intraperitoneal injection of ketamine $(100 \mathrm{mg} / \mathrm{kg})$ and xylazine $(10 \mathrm{mg} / \mathrm{kg})$. Femoral vein catheterization was performed. During imaging, animals were anesthetized with isoflurane/air using $1.0-1.5 \%$ via a nose cone with respiratory monitoring. Microbubbles were first warmed slowly to room temperature. Resuspension was performed until a homogenous milky-white suspension formed. For each imaging session, $0.2 \mathrm{~mL}$ of microbubble suspension (of $\sim 4 \%$ volume fraction for AMBs and $\sim 3.5 \%$ volume fraction for SonoVue ${ }^{\circledR}$ ) was slowly injected (over $\sim 10 \mathrm{~s}$ ) into femoral vein at a rate of $1.2 \mathrm{~mL} / \mathrm{min}$ to avoid possible microbubble destruction due to high pressure and shear stress.

\section{Susceptibility Contrast Imaging of Rat Brain}

In vivo brain imaging was performed in rats $(N=5)$ using a 38 -mm quadrature resonator. Anatomical images were acquired with two-dimensional (2D) fast low-angle shot (FLASH) sequence. Dynamic susceptibility weighted MRI was performed with single-shot GE echo-planner imaging (GE-EPI) sequence using TR $=1000 \mathrm{~ms}, \mathrm{TE}=30 \mathrm{~ms}$, flip angle $=90^{\circ}$, acquisition matrix $=96 \times 96$, spatial resolution $=$ $0.3 \times 0.3 \times 0.7 \mathrm{~mm}^{3}$ and NEX $=1$. Microbubble suspension was injected about $5 \mathrm{~min}$ after the start of dynamic imaging. A minimum lapse of $10 \mathrm{~min}$ was used to ensure sufficient clearance of the microbubbles before the next injection. The susceptibility effect of microbubbles was compared with that of MIONs [18-20] in brain by a single dose of $0.6 \mathrm{mg} \mathrm{Fe} / \mathrm{kg}$ injection using identical injection protocol and imaging sequence.

\section{Susceptibility Contrast Imaging of Rat Liver}

Liver imaging was performed in rats $(N=5)$ using a $60-\mathrm{mm}$ quadrature resonator. Dynamic MRI was then performed with respiratory-gated of similar protocol as brain imaging, except that $\mathrm{TE}=10 \mathrm{~ms}$, acquisition matrix $=64 \times 64$, spatial resolution $=0.78 \times 0.78 \times 2.0 \mathrm{~mm}^{3}$ and NEX $=1$. The susceptibility effect of microbubbles was also compared with that of MIONs in liver by a single dose of $0.6 \mathrm{mg} \mathrm{Fe} / \mathrm{kg}$ injection.

\section{E. In vivo Data Analysis}

GE-EPI images were first co-registered using AIR5.2.5 [21]. Apparent transverse relaxation rate enhancement $\left(\Delta R_{2}{ }^{*}\right)$ maps were computed on a pixel-by-pixel basis as $\Delta R_{2}^{*}=\ln$ $\left(\mathrm{S}_{\text {pre }} / \mathrm{S}_{\text {avg-post }}\right) / T E$ [22]. Assuming that $\Delta R_{2}{ }^{*}$ is proportional to microbubble concentration $\mathrm{C}(\mathrm{t})$ at time $\mathrm{t}, \mathrm{C}(\mathrm{t})$ can be estimated as $\mathrm{C}(\mathrm{t})=\mathrm{k} \ln \left\{\mathrm{S}_{\mathrm{pre}} / \mathrm{S}(\mathrm{t})\right\} / \mathrm{TE}+\mathrm{C}_{\mathrm{B}}$, where $\mathrm{S}(\mathrm{t})$ is the image intensity at time $t, k$ a proportionality constant, and $C_{B}$ a constant residue to account for any postinjection baseline [11]. Given the relatively long injection time and the limited lifetime of microbubbles in vivo, $\mathrm{C}(\mathrm{t})$ were approximately modeled with a gamma-variate function by curve fitting [23]. Full width at half maximum (FWHM) and time-to-peak were then measured from the fitted $\mathrm{C}(\mathrm{t})$ time courses.

\section{F. Ultrasound Demonstration}

Ultrasound imaging (Sonix RP, Ultrasonix) was preformed in rat livers $(N=2)$ using SonoVue ${ }^{\circledR}$ to demonstrate microbubble induced contrast change for comparison.

\section{G. Measurement of $\triangle R 2 *$ of $M I O N$-free and \\ MION-entrapped AMB and PMB Suspensions}

Microbubble phantom study was performed with 38-mm quadrature resonator. AMBs were diluted from a well-mixed microbubble suspension to $4 \%$ volume fraction with the addition of saline, while PMBs of $5 \%$ volume farction were prepared by addition of saline. The microbubbles were then placed in separate 2-mL cylindrical phantom tubes. Each phantom tube was slowly warmed to room temperature and gently mixed for 2 min outside the magnet prior to MR measurements. To ensure uniform suspension of microbubbles, the phantom was then continuously stirred by rotation inside the magnet. It was then arrested in horizontal position immediately before the start of MR acquisition sequence.

$\Delta R_{2}^{*}$ was measured by acquiring multi-echo GE signals continuously without phase encoding for $2 \mathrm{~min}$ from an axial 1 -mm slice at middle of the phantom as previously described [17]. Microbubble induced $\Delta R_{2}{ }^{*}$ was then calculated as the difference between $R_{2}{ }^{*}$ in the initial state and that in the final state. To demonstrate that MION were embedded and entrapped, microbubble-free suspending solutions $R_{2}{ }^{*}$ was measured before and after cavitation (using ultrasound of frequency $40 \mathrm{kHz}$ ) with multiple gradient echo sequences.

\section{RESUlts AND DisCUSSIONS}

\section{A. Characterization of AMBs and SonoVue ${ }^{\circledR}$}

The light micrographs of a representative batch of AMBs and SonoVue ${ }^{\circledR}$ microbubbles were depicted in Figs. 1(a) and (b), respectively. The estimated size distribution was from 1 to 23 $\mu \mathrm{m}$ (with $9.21 \mu \mathrm{m}$ mean diameter) for AMBs and 1 to $10 \mu \mathrm{m}$ (with $2.95 \mu \mathrm{m}$ mean diameter) for SonoVue ${ }^{\circledR}$. Fig. 2 shows the dependency of $\Delta R_{2}{ }^{*}$ on microbubble volume fractions in $\mathrm{AMBs}$ and SonoVue ${ }^{\circledR}$ microbubble suspension phantoms. An approximately linear relationship was observed. The relaxivities estimated from the fitted slopes were $58.52 \mathrm{(s}$. volume fraction $)^{-1}$ and $52.94(\mathrm{~s} \cdot \text { volume fraction })^{-1}$ for AMBs and SonoVue ${ }^{\circledR}$, respectively, consistent with the microbubble susceptibility effect in a theoretical study [9].

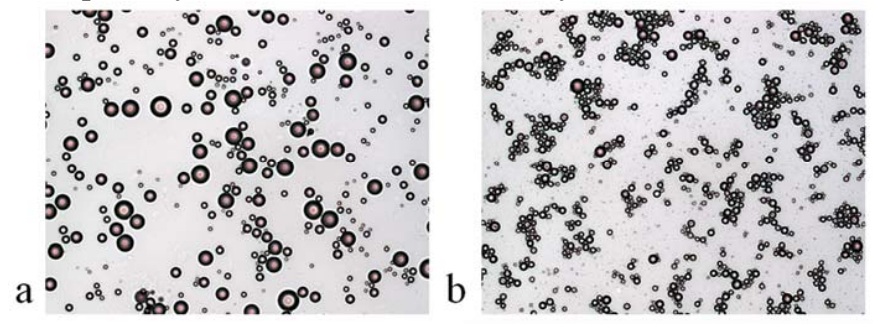

Fig. 1. Representative light micrograph (magnification $=20 x$ ) of (a)

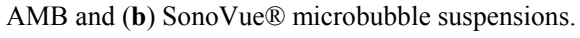



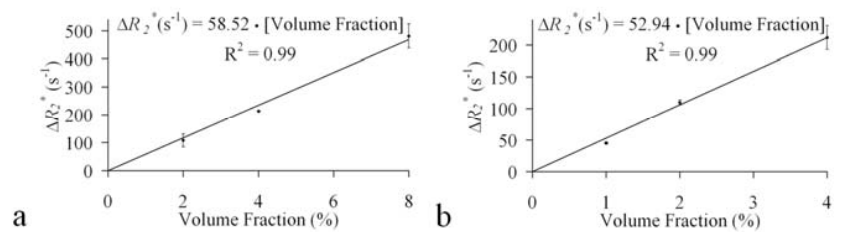

Fig. 2. Measured $\Delta R_{2}{ }^{*}$ of (a) AMBs and (b) SonoVue ${ }^{\circledR}$ microbubble suspensions versus microbubble volume fractions. The error bars represent standard deviation.

\section{B. In vivo Rat Brain Imaging}

Fig. 3 illustrates the rat brain images typically observed during AMBs injection. Note that the microbubbles induced $\Delta R_{2}{ }^{*}$ maps were observed to be similar to those caused by intravascular MION. Similar results were observed for SonoVue ${ }^{\circledR}$ injection. Table 1 shows the in vivo measurements of $\Delta R_{2}{ }^{*}$, FWHM and time-to-peak of AMBs and SonoVue ${ }^{\circledR}$, as well as $\Delta R_{2}^{*}$ and time-to-peak of MION in cortex area among all rats studied. Time-to-peak of microbubbles was found to be longer than that of MION. This is largely expected as microbubbles, with size comparable to that of red blood cells, flow slower than blood plasma while MION nanoparticles flow together with plasma. In few of the rats studied ( 1 out of 5 in brain imaging), the $T_{2}{ }^{*}$-weighted signals after microbubble injection did not return to the preinjection baseline, this may be caused by microbubble trapping in local tissue vasculature.

a
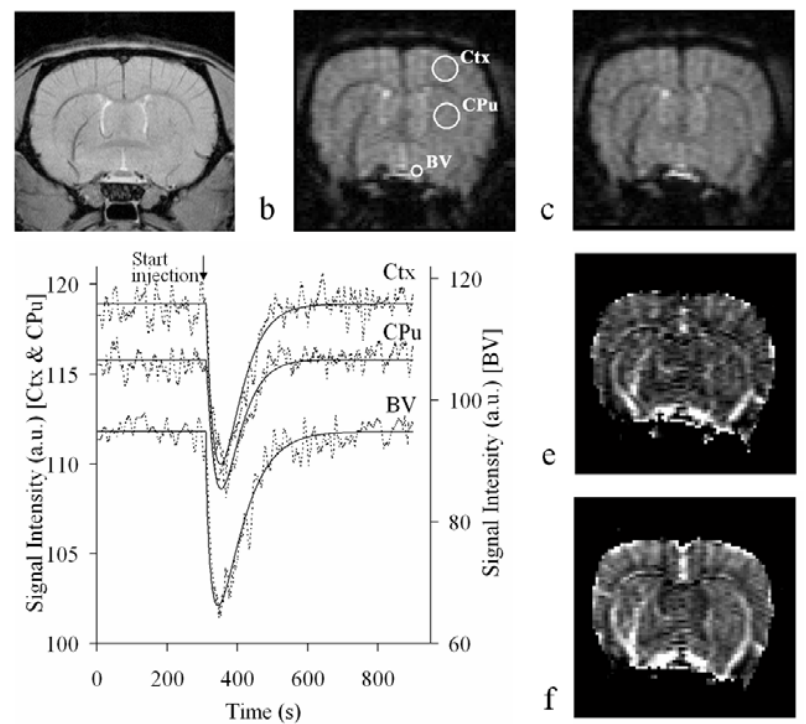

Fig. 3. Typical images from a rat brain during AMBs injection: (a) anatomical image; (b) preinjection GE-EPI $T_{2}{ }^{*}$-weighted image; (c) postinjection GE-EPI $T_{2}{ }^{*}$-weighted image with the maximum susceptibility contrast; (d) $T_{2}{ }^{*}$-weighted signal time courses in different ROIs during AMBs injection; (e) $\Delta R_{2}{ }^{*}$ map computed from AMB data; and (f) $\Delta R_{2}{ }^{*}$ map obtained using intravascular contrast agent MIONs at $0.6 \mathrm{mg} \mathrm{Fe} / \mathrm{kg}$. Three ROIs used for time course measurements are shown in (b) and gamma-variate fitted data shown in sold lines.

\section{In vivo Rat Liver Imaging}

Fig. 4 illustrates the rat liver images typically observed during

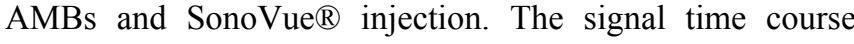
during SonoVue $\AA$ injection captured by ultrasound imaging was overlaid in grey for comparison. Note that the ultrasound signal was enhanced by microbubbles. Similar signal recovering patterns were observed, showing comparable lifetime of SonoVue ${ }^{\circledR}$ in vivo. Table 1 shows the measured $\Delta R_{2}{ }^{*}$, FWHM and time-to-peak of AMBs and SonoVue ${ }^{\circledR}$, as well as $\Delta R_{2}{ }^{*}$ and time-to-peak of MION in liver. Time-to-peak of microbubbles was also found to be longer than that of MION. In few of the rats studied ( 2 out of 5 in liver imaging), the $T_{2}{ }^{*}$-weighted signals after microbubble injection did not return to the preinjection baseline, possible uptake of intact microbubbles by Kupffer cells in liver may also contribute to such observation.

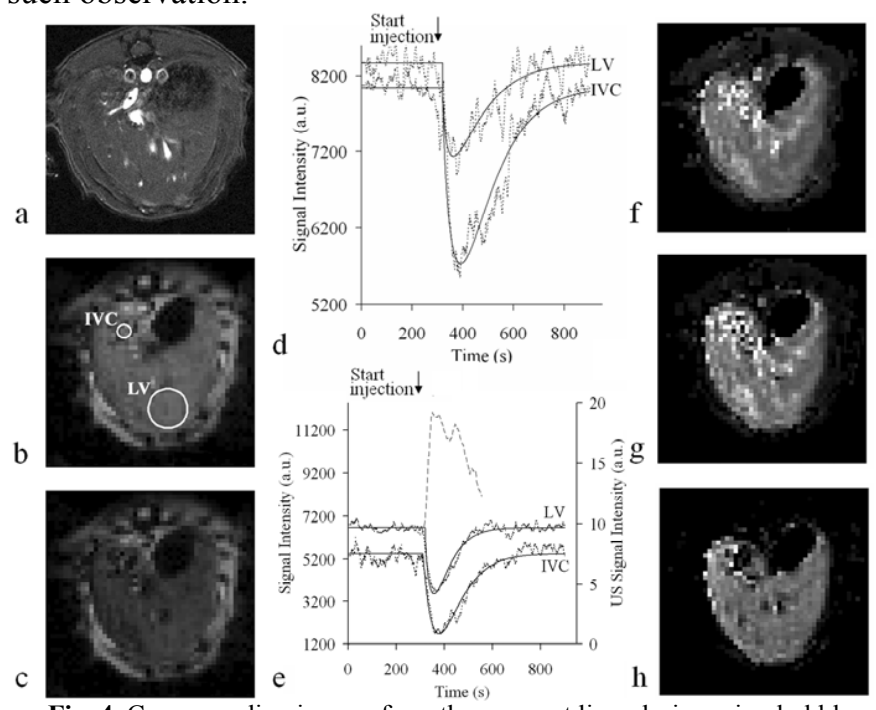

Fig. 4. Corresponding images from the same rat liver during microbubble injections: (a) anatomical image, (b) preinjection GE-EPI $T_{2}{ }^{*}$-weighted image for AMBs and (c) postinjection GE-EPI $T_{2}{ }^{*}$-weighted image with the maximum susceptibility contrast for AMB. $T_{2}{ }^{*}$-weighted signal time courses in different liver regions during (d) AMB and (e) SonoVue ${ }^{\circledR}$ injection in the same rat. $\Delta R_{2}{ }^{*}$ maps for (f) AMBs, (g) SonoVue ${ }^{\circledR}$ and (h) $0.6 \mathrm{mg} \mathrm{Fe} / \mathrm{kg} \mathrm{MIONs}$. Two ROIs for time course measurement, a homogenous liver region (LV) and the region covering inferior vena cava (IVC), are shown in (b). Gamma-variate fitted data are shown in sold lines. Ultrasound measurement (grey line) was overlaid in (e) for comparison.

Table 1. In vivo measurements of relaxation rate enhancement $\left(\Delta R_{2}{ }^{*}\right)$, full width at half maximum (FWHM) and time-to-peak of the concentration time courses for AMBs, SonoVue ${ }^{\circledR}$ and MIONs in rat brain cortex (mean \pm standard deviation, $N=5$ ) and liver (mean \pm standard deviation, $N=5$ ).

\begin{tabular}{lccc}
\hline & $\Delta R_{2}^{*}\left(\mathrm{~s}^{-1}\right)$ & FWHM (s) & Time-to-peak (s) \\
\hline Brain & & & \\
AMBs & $2.49 \pm 1.00$ & $114 \pm 39$ & $57 \pm 20$ \\
SonoVue ${ }^{2}$ & $2.41 \pm 1.18$ & $86 \pm 16$ & $48 \pm 12$ \\
MIONs & $1.98 \pm 0.36$ & N.A. & $24 \pm 2$ \\
& & & \\
Liver & & & \\
AMBs & $40.89 \pm 22.08$ & $119 \pm 68$ & $60 \pm 24$ \\
SonoVue & $45.78 \pm 34.22$ & $95 \pm 41$ & $58 \pm 28$ \\
MIONs & $55.84 \pm 21.75$ & N.A. & $39 \pm 14$ \\
\hline
\end{tabular}

D. Measurement of $\triangle R 2 *$ of MION-free and MION-entrapped PMB Suspensions

Fig. 5 shows the individual microbubble-induced $\Delta R_{2}{ }^{*}$ values in six batches of PMBs at $5 \%$ volume fraction and one batch of AMBs at $4 \%$ volume fraction with and without MIONs, each measured with six repeated measurements. Suspending solution $R_{2}{ }^{*}$ for AMBs without and with MIONs after cavitation were found to be increased by $1.41 \mathrm{~s}^{-1}$ and $23.91 \mathrm{~s}^{-1}$ respectively. Similarly, suspending solution $R_{2}{ }^{*}$ for PMBs 
without and with MIONs after cavitation were found to be increased by $0.3 \pm 0.4 \mathrm{~s}^{-1}$ and $55 \pm 9 \mathrm{~s}^{-1}$ respectively. These substantial differences for microbubbles with MIONs demonstrate that there were more MIONs in the suspending solution after cavitation; suggesting that embedded and entrapped MION were released into suspending solution after the microbubble cavitation. Transmission electron microscopy was done on AMBs with MION and depicted in Fig. 6. MIONs as dark dots (red arrows) were observed on the shells of AMB, validating MIONs were embedded onto shells of AMBs. Nevertheless, small amount of free MIONs were also observed in suspending solution (green arrow). Larger iron oxide nanoparticles like superparamagnetic iron oxide nanoparticles (SPIOs) or $\mathrm{FePt} @ \mathrm{Fe}_{2} \mathrm{O}_{3}$ yolk-shell nanoparticles [24], may be used to further enhance the microbubble magnetic susceptibility effect [25].

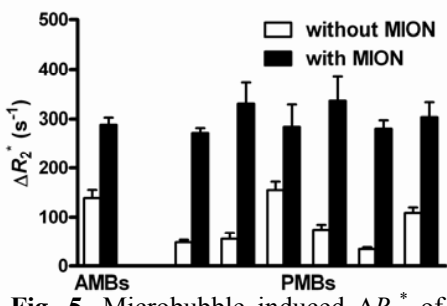

Fig. 5. Microbubble induced $\Delta R_{2}{ }^{*}$ of Fig. 6. Transmission electron different microbubbles. The error bars microsgraph shows MIONs were represent one standard deviation embedded onto shells of AMBs

\section{CONCLUSIONS}

In this study, we investigated the feasibility of gas-filled microbubbles as an intravascular MR susceptibility contrast agent in vivo at $7 \mathrm{~T}$. Considerable susceptibility induced changes were observed and characterized in rat brain and liver using the custom-made albumin-coated microbubbles and a commercially available clinical ultrasound microbubble contrast agent. The results indicate that microbubbles can serve as a unique intravascular MR contrast agent at high field. We also demonstrated, for the first time, that embedding or entrapping iron oxide nanoparticles in microbubbles is feasible. With such approach, microbubble susceptibility can be significantly enhanced, so that microbubbles can be monitored with high sensitivity and low concentrations under MRI. Such capability has the potential to lead to real-time MRI guidance in various microbubble-based drug delivery and therapeutic applications.

\section{ACKNOWLEDGMENT}

We thank Dr. Joseph C.K. Leung in the Department of Medicine, Dr. Danny Chan in the Department of Biochemistry and Dr. Ke Xia Cai at the Laboratory of Biomedical Imaging and Signal Processing of The University of Hong Kong for assistance. This work was supported by the Hong Kong Research Grant Council (CERG HKU 7642/06M).

\section{REFERENCES}

[1] A. Bouakaz, M. Versluis, and N. de Jong, "High-speed optical observations of contrast agent destruction," Ultrasound in Medicine \& Biology, vol. 31, pp. 391-9, 2005.

[2] E. C. Unger, E. Hersh, M. Vannan, T. O. Matsunaga, and T. McCreery,
"Local drug and gene delivery through microbubbles," Prog Cardiovasc Dis, vol. 44, pp. 45-54, Jul-Aug 2001.

[3] S. Mehier-Humbert, T. Bettinger, F. Yan, and R. H. Guy, "Plasma membrane poration induced by ultrasound exposure: implication for drug delivery," J Control Release, vol. 104, pp. 213-22, May 52005.

[4] R. Bekeredjian, H. A. Katus, and H. F. Kuecherer, "Therapeutic use of ultrasound targeted microbubble destruction: a review of non-cardiac applications," Ultraschall Med, vol. 27, pp. 134-40, Apr 2006.

[5] W. C. Culp, T. R. Porter, J. Lowery, F. Xie, P. K. Roberson, and L. Marky, "Intracranial clot lysis with intravenous microbubbles and transcranial ultrasound in swine," Stroke, vol. 35, pp. 2407-11, 2004.

[6] K. Hynynen, N. McDannold, N. A. Sheikov, F. A. Jolesz, and N. Vykhodtseva, "Local and reversible blood-brain barrier disruption by noninvasive focused ultrasound at frequencies suitable for trans-skull sonications," Neuroimage, vol. 24, pp. 12-20, 2005.

[7] Y. Kaneko, T. Maruyama, K. Takegami, T. Watanabe, H. Mitsui, K Hanajiri, H. Nagawa, and Y. Matsumoto, "Use of a microbubble agent to increase the effects of high intensity focused ultrasound on liver tissue," European Radiology, vol. 15, pp. 1415-20, 2005.

[8] M. E. Moseley, M. F. Wendland, I. Rampil, and J. Barnhart, "Microbubbles: a novel MR susceptibility contrast agent," in Proceedings of the 10th Annual Meeting of the ISMRM, San Francisco, California, USA, 1991, p. 1020.

[9] R. Dharmakumar, D. B. Plewes, and G. A. Wright, "On the parameters affecting the sensitivity of MR measures of pressure with microbubbles," Magn Reson Med, vol. 47, pp. 264-73, Feb 2002.

[10] A. L. Alexander, T. T. McCreery, T. R. Barrette, A. F. Gmitro, and E. C. Unger, "Microbubbles as novel pressure-sensitive MR contrast agents," Magnetic Resonance in Medicine, vol. 35, pp. 801-6, 1996.

[11] K. K. Wong, I. Huang, Y. R. Kim, H. Tang, E. S. Yang, K. K. Kwong, and E. X. Wu, "In vivo study of microbubbles as an MR susceptibility contrast agent," Magn Reson Med, vol. 52, pp. 445-52, Sep 2004.

[12] T. Ueguchi, Y. Tanaka, S. Hamada, R. Kawamoto, Y. Ogata, M. Matsumoto, H. Nakamura, and T. Johkoh, "Air microbubbles as MR susceptibility contrast agent at 1.5 Tesla," Magn Reson Med Sci, vol. 5 , pp. 147-50, Oct 2006.

[13] F. De Guio, H. Benoit-Cattin, and A. Davenel, "Signal decay due to susceptibility-induced intravoxel dephasing on multiple air-filled cylinders: MRI simulations and experiments," Magma, vol. 21, pp. 261-71, Jul 2008

[14] J. S. Cheung, A. M. Chow, H. Guo, and E. X. Wu, "Microbubbles as a novel contrast agent for brain MRI," Neuroimage, vol. 46, pp. 658-64, Jul 12009.

[15] E. X. Wu, H. Tang, and J. H. Jensen, "Applications of ultrasmall superparamagnetic iron oxide contrast agents in the MR study of animal models," NMR Biomed, vol. 17, pp. 478-83, Nov 2004.

[16] D. Cerny, G. J. Mills, and P. J. Westkaemper, "Continuous Sonication Method for Preparation Protein Encapculated Microbubbles," United States, 1990.

[17] A. Chow, J. Cheung, and E. Weu, "Enhancement of Gas-filled Microbubble Magnetic Susceptibility by Iron Oxide Nanoparticles," in Proceedings of the 17th Annual Meeting of the ISMRM, Honolulu, Hawaii, USA, 2009, p. 1486.

[18] E. X. Wu, K. K. Wong, M. Andrassy, and H. Tang, "High-resolution in vivo CBV mapping with MRI in wild-type mice," Magn Reson Med, vol. 49, pp. 765-70, Apr 2003.

[19] E. X. Wu, H. Tang, and J. H. Jensen, "High-resolution MR imaging of mouse brain microvasculature using the relaxation rate shift index $\mathrm{Q}, "$ NMR Biomed, vol. 17, pp. 507-12, Nov 2004.

[20] E. X. Wu, H. Tang, T. Asai, and S. D. Yan, "Regional cerebral blood volume reduction in transgenic mutant APP (V717F, K670N/M671L) mice," Neurosci Lett, vol. 365, pp. 223-7, Jul 292004.

[21] R. P. Woods, S. T. Grafton, C. J. Holmes, S. R. Cherry, and J. C. Mazziotta, "Automated image registration: I. General methods and intrasubject, intramodality validation," J Comput Assist Tomogr, vol. 22, pp. 139-52, Jan-Feb 1998.

[22] E. X. Wu, H. Tang, K. K. Wong, and J. Wang, "Mapping cyclic change of regional myocardial blood volume using steady-state susceptibility effect of iron oxide nanoparticles," J Magn Reson Imaging, vol. 19, pp. 50-8, Jan 2004

[23] D. R. Pickens, "Perfusion/diffusion quantitation with magnetic resonance imaging," Invest Radiol, vol. 27 Suppl 2, pp. S12-7, Dec 1992.

[24] J. Gao, G. Liang, J. S. Cheung, Y. Pan, Y. Kuang, F. Zhao, B. Zhang, X. Zhang, E. X. Wu, and B. Xu, "Multifunctional yolk-shell nanoparticles: a potential MRI contrast and anticancer agent," J Am Chem Soc, vol. 130, pp. 11828-33, Sep 32008.

[25] R. Dharmakumar, D. B. Plewes, and G. A. Wright, "A novel microbubble construct for intracardiac or intravascular MR manometry: a theoretical study," Phys Med Biol, vol. 50, pp. 4745-62, Oct 212005. 